\title{
Utilisation de la calculatrice électronique pour l'étude des oscillations du système des galeries d'amenée de l'aménagement de Saint-Martin-Vésubie
}

\author{
Use of an electronic calculator for studying \\ the oscillations of the supply tunnel system \\ in the St. Martin-Vésubie project
}

\begin{abstract}
Liordinateur électronique a permis de calculer les oscillations des plans d'eau dans le système puits-cheminée d'équilibre de Saint-MartinVésubie. La souplesse du modèle mathématique utilisé a permis de comparer différentes solutions, correspondant a certaines valeurs des parametres, dans des conditions de prix et de délais très avantageux.
\end{abstract}

\begin{abstract}
The ase of en electronic ordinator made it possible to calculate lac uater level ascillulions in the shaft and surge tank system of the st. Marlin-Vesubie project The flexibility of the mathemulical model used enabled a number of different solutions corresponding to certain parameter values to be compared and favourable price and delivery conditions to be maintained.
\end{abstract}

\section{I. - POSITION DU PROBLÈME}

L'aménagement projeté à Saint-Martin-Vésubie comportera, dans le stade de réalisation finale, une galerie principale $A B$ reliant le réservoir d'accumulation de Giricopion à l'extrémité amont de la conduite forcée. Sur cette galerie sont greffées en D l'adduction du torrent «La Madone des Fenêtres » au moyen du puits incliné CD et en $\mathrm{E}$ l'adduction de deux autres torrents: le Spaillard et la Gordolasque. Cette deuxième adduction comprend la galerie EF, le puits FG, placé au droit de la prise du Spaillard, et la galerie à écoulement libre ${ }_{\mathrm{GH}} \mathrm{H}$, transitant les eaux de la Gordolasque vers le puits GF.
L'ensemble de ce système d'amenée sera le siège, en période transitoire, d'oscillations complexes, les deux puits jouant le rôle de deux cheminées d'équilibre supplémentaires.

E.D.F. Alpes III ayant demandé à la SogreAH d'analyser les régimes d'oscillation auxquels on peut s'attendre en exploitation et, en particulier, d'examiner les temps de manouvre les plus défavorables, il a été décidé d'utiliser dans ce but la machine électronique IBM du Service scientifique de notre Société.

\section{II. - PRINCIPE DU CALCUL}

Les équations du mouvement transitoire dans les systèmes de conduites sont bien comnues. Ce sont:

$1^{\circ}$ Les équations dynamiques de la forme :

$$
\frac{\mathrm{L}}{g l} \quad \frac{d \mathrm{Q}}{d t}+\mathrm{Z}+\mathrm{H}=0
$$

$Z$ étant la différence des hauteurs piézométriqutes entre l'aval et l'amont du tronçon des conduites considérées.

$H$ étant la perte de charge $\left(H=k Q^{2}\right)-$ les autres notations étant classiques. 


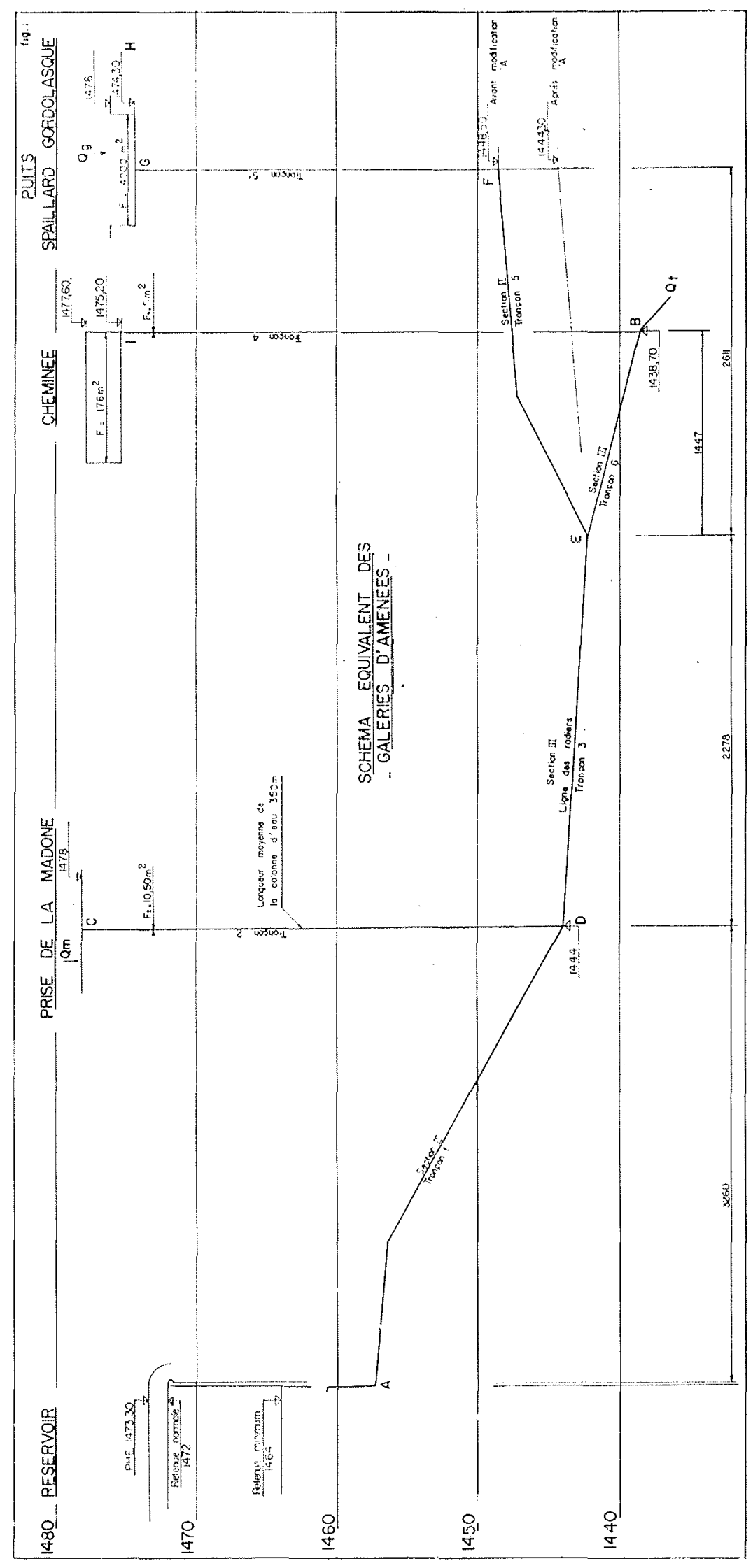

2. Les équations de continuité.

Pour les noeuds, elles sont de la forme:

$$
Q_{1}+Q_{2}+Q_{3}=0
$$

Pour les surfaces libres, elles sont de la forme :

$$
\mathrm{Q}=\mathrm{F} \frac{d z}{d t}
$$

F : section horizontale du puils de cheminée:

$z=$ cote de la surface libre.

Le calcul fait repose sur certaines hypothèses simplificatrices :

- On ne tient pas comple de l'inertie dans les troncons courts à faible vitesse : puits FG et chemince principale;

- Par contre, il a été nécessaire d'en tenir comple dans le puits GD, la vitesse de l'eau $\mathrm{y}$ ćtant relativement forte.

On a, d'autre part, admis que la galerie à surface libre $\mathrm{GH}$ se comporte simplement comme une expansion très grande, les phénomènes transitoires pouvant y prendre naissance ne devant pas affecter de manière sensible le régime d'oscillation dans l'ensemble de l'installation.

Ces hypothèses ne sont faites que pour réduire les temps de calculs, elles sont compatibles avec la précision demandée. On pourrait très bien envisager de traiter le cas général sur l'ordinateur.

On est ainsi amené à résoudre un système différentiel de 11 équations à 11 inconnues (5́ équations dynamiques et 6 équations de continuité).

La méthode de calcul utilisée est une méthode d'intégration classique par différences finies.

Dans le cas présent, il était de toute facon impensable d'envisager une solution à la main, le Maitre de l'Cuvre demandant que cette étude soit faite très rapidement, étant donné l'état d'avancement des travaux, en particulier dans la cheminée d'équilibre.

\section{III. - RÉSULTATS}

La machine a permis de traiter dans le délai imparti plus de vingt cas de manœuvre grâce à un programme de calcul standard.

Cette étude a fait apparaître la nécessité d'apporter quelques modifications à l'installation projetée, en particulier à cause de l'amplitude des oscillations constatées dans le puits FG. Nous 
Fig. 1

Chemines d'épuilibre

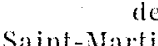
c a $4 \mathrm{~m}^{\prime \prime}$ on 8 s et ouverture 4 a $8 \mathrm{~m} \% \mathrm{~s}$ 'n $8 \mathrm{~s}$ apres 64 secondes bébit non reparti Niveatu slatique 1464 Galeries revetues a $85 \%$
FIG. 22

Cheminé d'équilibre Saint-Marlin-Vesubic Onverture $O$ at $+111 \% / \mathrm{s}$ en 8 s ct ouverture it a $8 \mathrm{~m}^{3} / \mathrm{s}$ en $8 \mathrm{~s}$ après 64 secondes Débit non réparti Vircan statique 1464 Expansion $\mathrm{S}=30 \mathrm{~m}=$ au-dessous de la cote 1456 Galeries revêtues a 85
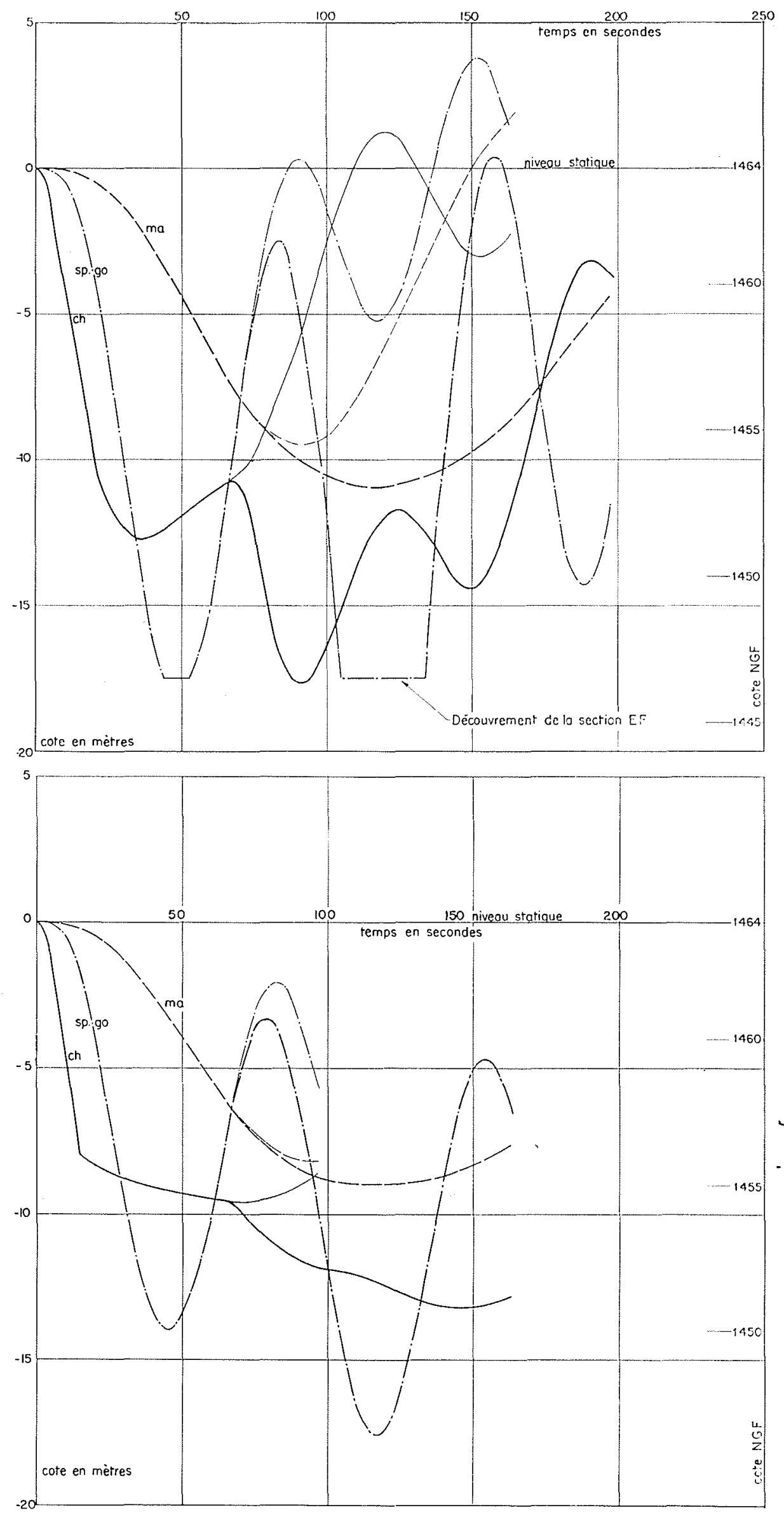
avons proposé d'abaisser la cote du point $\mathrm{F}$ et, de plus, d'implanter à la partie inférieure de la cheminée principale une petite chambre d'expansion, dans le but de supprimer les decouvre- ments de la galerie EF, dans la partie onest. Les deux graphiques font apparaître l'influence de cette expansion sur l'amplitude des oscillations dans le puits $F G$.

\section{IV. - CONCLUSION}

L'utilisation des machines électroniques permet de résoudre facilement et rapidement des problèmes qui demandaient jusqu'alors un travail extremement long et fastidieux, a condition cependant - et ceci est très important - que l'on puisse réunir autour de ces machines les spécialistes de la programmation et de la technique du problème traité.
Il est d'autre part toujours possible de conserver les programmes ou sous-programmes de calcul, de sorte que s'il se présente par la suite un problème analogue à résondre, le travail de préparation est fortement réduit.

M. PACCARD, (INGBietir a ha sogreah).

\section{NO'TRE FRON'IISPICE}

HEL,MHOLTZ (1821-1894)

Dès sa jrime jeunesse, Helmboltz se sentit vivenent attire par la physique; mais sa famille fit de lui un médecin militaire, carrière qui semblait plus sure et plus rémunératrice..

Hermann Ludwig Ferdinand von Helmholtz ctatit né le 31 aout 1821 a Potsdan, où son père était professeur au « gymnatse ». Doctettr en médecine, puis médecin militaire à Potsdan en. 1843 , il ne tarda pas à domer sa mesure dans son célèbre mémoire Ueber die Erhaltung der Kraft (Sur la conservation de la force, nous dirions aujourd'hui : de l'anergio) (1847), qui tit époque, et traça les voies de l'énergéticiue noderne.

Nommé professeur d'anatomie et physiologie à Konigsber (1849) puis à Bonn (1855) et à Heidelberg (1858), il put donner peu a peu libre cours a ses inclinations dantan en orientant ses travaux sur l'acoustique et la théorie de la musiquc qu'il allait totalement renouveler, sur fopticue physiolorique cui le conduisit au processus d accommodation du cristallin et en 1851, à l'invention de Pophtalmoscope, sur l'hydrodynanique, discinine dans laquelle il eturlia les mouvements discontinus de l'eau et découvrit dès 1858 les lois fondamentales de 1 . theorie des tolithilons. Ses recherches de 1866-68: « Sur les fondements de la Géometrie », soulignent les origines empiriques de cette science et préparent les voies it la Relativité et aux Quanta.

En $1870-71$, nous le retrouvons professen de physique theorique a Berlin; là, il toume su attention vers la jetne electricité, applique les lois de la thermodynamicue à la théorie de la pile electrique, donne la notion de couche double électrique et démontre l'existence d'un atonce d'électricité qui revait devenir l'électron. Avec Gibbs, il fut un des fondiateurs de la thermo. iynamique chimique; il donna le premier une théric com plete de la dispersion de la lumière par la matière.

L'expérimentation l'avait conduit à lat philosoplue, lui faisant rejeter les idées préconçues et proclamer que « toute connaissance est fondee sur l'expericnce, transmise par héritayc on sance est

Une cuvre aussi riche, aussi delectique et ausi féconde le fait

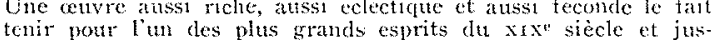
tenir pon lan des plus grands esprits du xix" siecle et jus-
tifie lopinion selon lacutelle " il domina presque tonte lat science de son époqute

Felmholtz mourut it Charlottenbourg le 8 septenbre 1894 alors yu'il ctait directeur de la Technische Reichanstalt baboratoire physico-technique) dont il ititit le fonditeur.
HELMHOLTZ (1821.189\%)

Helmholte folt a strong attration to physics when he was wait young but his family made him become an army doctor as tha scemed to be a safor and better paid carecr.

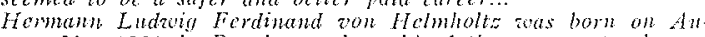
yikst $31 \mathrm{st} 1821$ in Potsdam, where his father wots a teacher a the "Crymasium." After gualifying and becomin! an army doctor at Potsdam in 1843, it ails not long before he demon strated true abilities with his epoch making Veber die Erhaltung der Kraft, (On the conservation of force, or. as aic would say today, energy) (1847) which pawed the way for modern cuergetics.

He was appoinled Professor of Anatomy and Physiology a Kanigsberg (1849), then at Bonn (1855) and at Heidelber (1858), and was thus gradually able to give frec roin to his carlier inclinations by directing his atention to aconstios and the theory of music zehich he completcly rezised, to physiologi cal optics which led him to his explanation of the mechanism of "ccommodation of the cye's chrystallinc lens and, in 1851, to his ingention of the ophthalmoscope. Ho also concened himself with hydrodynanics and intestiguted the discontimus motion of water. In 1858 he discovered the fundamental lawes of the Hicory of vortices. His work from 1866 to 1868 , alhich resmited in his paper, On the randamentats of Geometry, miderline

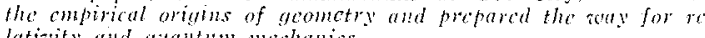
lativity and gitamin mechanics.

In $1870-71$ he became Professor of theuretical physics at Bir lin where he turned to lectricits. He applicd the lases of the modynames to the electre battery, formulated the concept of the clectrical double layer and proved the existence of an ele the atom. atcr to become the electron. Fe and Gibbs founded chemical thermodynamics betacen then and he ains the firs? person to formulate a complete theory oxplaining the dispersion of light by matter.

His cxperinental awe led him to philosophy. He rejected all proconceined ideas and proclaimed that "all knowledie is

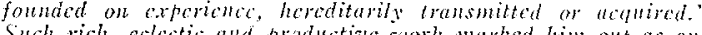

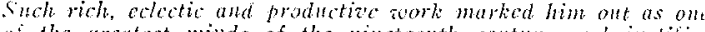
of the gratest minds of the nincteenth contury and jistific the opinion that "he dominated nealy all the scienec of hi

Helmholtz died at Charlotentury on September Sth 1894, when he was director of the Technische Reichanstalt (Physico-tich 